\title{
Particulate air pollution and mortality in 38 of China's largest cities: time series analysis
}

The authors of this Research paper (BMJ 2017;356:j667, doi:10. $1136 / \mathrm{bmj} . j 667$ ) have informed us of two errors.

In the sentence, "A back-of-envelope calculation reveals that bringing China's PM10 level to the WHO

standard-20ug/m3-would save 3 million premature deaths each year," 3 million should read 0.3 million.
The affiliation for Tong Liu and Yuhang Pan should read, "Division of Environment, The Hong Kong University of Science and Technology, Hong Kong, China."

These errors have now been rectified. 